\title{
Influence of the Solar Wind/Interplanetary Medium on Saturnian Kilometric Radiation
}

\author{
H. O. RUCKER ${ }^{1}$ and M. D. DESCH ${ }^{2}$ \\ ${ }^{1}$ Space Research Institute, Austrian Academy of Sciences, Lustbühel Observatory, A-8042 Graz, Austria \\ ${ }^{2}$ NASA/Goddard Space Flight Center, Laboratory for Extraterrestrial Physics, \\ Greenbelt, MD 20771, U.S.A. \\ (Received July 7, 1989) \\ (Updated version of a paper presented at IUGG, Hamburg, FRG, 1983)
}

\begin{abstract}
Previous studies on the periodicities of the Saturnian kilometric radiation (SKR) suggested a considerable solar wind influence on the occurrence of SKR, so it was obvious to investigate the relationship between parameters of the solar wind/interplanetary medium and this Saturnian radio component.

Voyager 2 data from the Plasma Science experiment, the Magnetometer experiment and the Planetary Radio Astronomy experiment were used to analyse the external control of SKR. Out of the examined quantities known to be important in controlling magnetospheric processes this investigation yielded a dominance of the solar wind momentum, ram pressure and kinetic energy flux, in stimulating SKR and controlling its activity and emitted energy, and confirmed the results of the Voyager 1 analysis.
\end{abstract}

\section{Introduction}

The search for the planetary radio emissions made an enormous step forward by the Voyager 1 and 2 observations of the Jovian and Saturnian nonthermal radiations. Voyager 2 continued the exploration of the planets Uranus and Neptune with the detection of characteristic radio emissions. Comparative studies on the properties of these radiations yielded that the Jovian radio spectrum is rather unique and that Saturn as a radio emitter is much more Earth-like than Jovian.

In the case of Saturn three nonthermal radio components were discovered by the Voyager mission: the Saturn kilometric radiation (SKR), narrowband noise and related continuum, and Saturn electrostatic discharges (SED) (KAISER et al., 1984), but only two components (SKR and SED) are freely propagating waves outside the Saturnian magnetosphere. The SED, a lightning-like emission from an atmospheric storm system (KAISER et al., 1983) could be observed only for a few days around both Voyager encounters with Saturn. On the contrary the SKR was measured by both spacecraft many months before encounter. Considering the relatively well known SKR source location within the Saturnian magnetosphere (KAISER and DESCH, 1982; LECACHEUX and GENOVA, 1983) and the long monitoring period around the encounters this radio emission provides a valuable tool for remote sensing of the magnetospheric conditions.

Since the days of investigating planetary radio emissions an increasing interest was laid on the interaction between the solar wind and the planetary magnetospheres, in particular on the influence, stimulation, or even control of the solar wind on planetary 
radiations. A number of papers have examined a possible relationship between the solar activity/solar wind and the Jovian decametric radiation (CONSEIL et al., 1971; KOV.ALENKO, 1971; BARROW, 1972, 1978, 1979; OYA and MORIOKA, 1977; TERASAWA et al., 1978) and other Jovian radio sources (DESCH and BARROW, 1984). Similar studies were carried out in searching for a close connection between the solar wind and the terrestrial radiation (GALLAGHER and D'ANGELO, 1981) and associated magnetospheric processes (KAISER and ALEXANDER, 1977; GREEN et al., 1979, 1982).

Since the source location of the Saturn kilometric radiation in the dayside high latitude cleft region suggested a direct access of the solar wind particles, studies on the external control on SKR were performed.

First evidence for solar wind control of SKR was provided by DESCH (1982), who found that the power level fluctuations of SKR strongly depend on the kinetic ram pressure variations. Furthermore the 25-day periodicity of the emitted SKR power was found to be consistent with the solar rotation period. While this investigation was based upon a semiquantitative measure of the SKR, which was given in hours of activity, a subsequent paper (DESCH and RUCKER, 1983) introduced an accurate quantitative measure of the radiated SKR wave energy, averaged over one Saturn rotation, and extended the examination to solar wind quantities incorporating interplanetary magnetic field (IMF) properties. This paper was still limited to Voyager 1 observations, but continuations (RUCKER and DESCH, 1983; DESCH and RUCKER, 1985; RUCKER et al., 1989) and the present paper treat the Voyager 2 spacecraft measurements.

Concerning the relationship between the Saturn kilometric radiation and the solar wind the Voyager 1 studies could apply the linear cross correlation procedure due to almost continuous time series of the solar wind, IMF and radio data. Unlike Voyager 1, the Voyager 2 spacecraft observed several times very low solar wind plasma density regions, in which the magnetic field orientation coincided with the Jovian magnetotail direction. As explained later on these indications brought evidence of a multiple immersion of the Voyager 2 spacecraft in the extended Jovian magnetotail and at the same time a screening off the "normal" solar wind conditions. In consequence of these circumstances the time series of the normal solar wind and IMF data have frequent interruptions. In order to deal with this type of data structure a different treatment, the so-called method of superposed epochs, was used to analyse the influence of the solar wind/interplanetary medium on the Saturnian kilometric radiation.

\section{Solar Wind, IMF, and SKR Data as Observed by Voyager 2}

This study makes use of data, which were obtained by three experiments onboard Voyager 2. The solar wind plasma data are from the Plasma Science (PLS) experiment, the interplanetary magnetic field data are from the Magnetometer (MAG) experiment, and the radio data are from the Planetary Radio Astronomy (PRA) experiment. This analysis covers the Saturn preencounter time interval from 1 January 1981 through 25 August 1981, one day before the closest approach of Voyager 2 at Saturn. As already explained in a previous paper (DESCH and RUCKER, 1983) the location of the SKR source in the dayside Saturnian cleft region implies the use of only preencounter data; after the encounter the spacecraft departs at the Saturnian nightside.

Figure 1 shows an overview of the radio, plasma, and magnetic field quantities over the preencounter period as mentioned above. The top panel of Fig. 1 shows the measured 

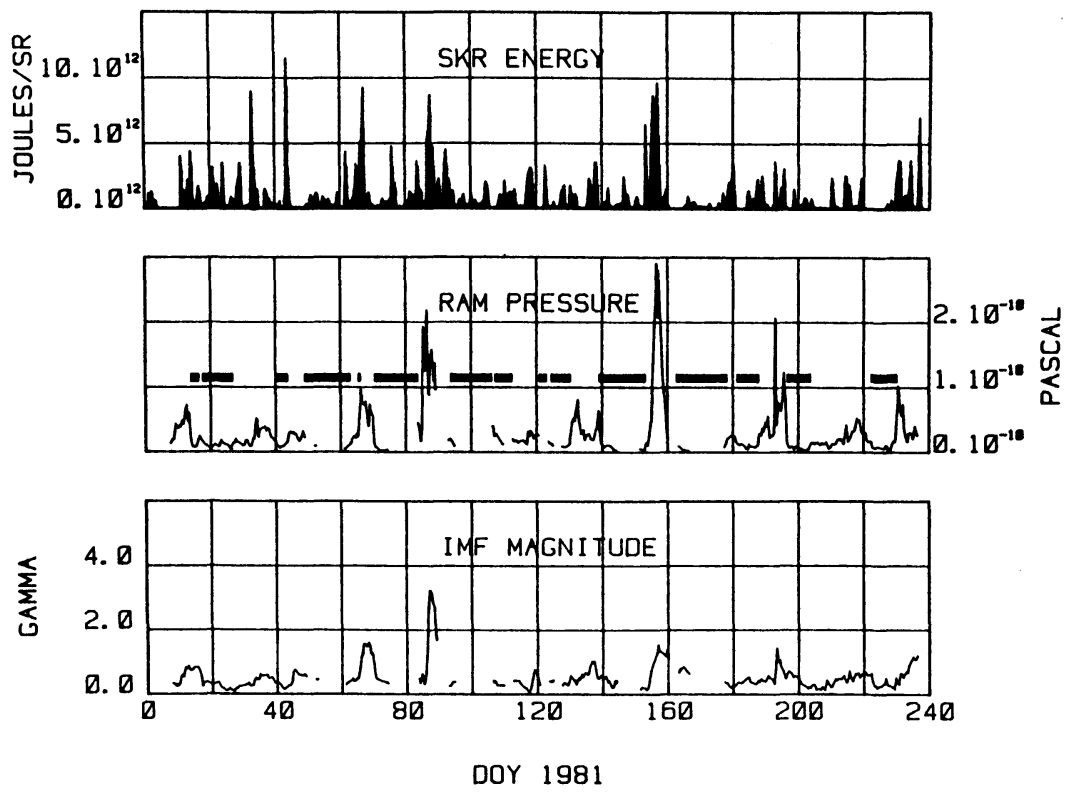

Fig. 1. Voyager 2 planetary radio astronomy, plasma science, and magnetometer data for the Saturn preencounter period in 1981. Solar wind ram pressure and interplanetary magnetic field (IMF) magnitude are compared with the Saturn kilometric radiation (SKR) energy per rotation at the Saturn interaction point. The bars in the ram pressure panel indicate the Jovian tail encounter periods.

SKR energy per Saturn rotation in units of Joules per steradian. In order to obtain the shown SKR energy values the 1-hour averaged SKR flux densities were integrated over the SKR emission bandwidth and then integrated over one Saturn rotation (10.66 hours). Only those values are considered, which exceeded a flux density of $1.3 \times 10^{-20} \mathrm{Wm}^{-2} \mathrm{~Hz}^{-1}$ at 1 AU. Comparing this SKR energy data set with the Voyager 1 measured SKR energy values a rough estimate yields a weaker SKR activity during the Voyager 2 preencounter period.

The second panel of Fig. 1 shows the solar wind ram pressure (density times speed squared), given in units of pascal. This solar wind quantity is already propagated from the point of observation to the point of interaction at the Saturnian magnetosphere. This "forward propagation" of solar wind features takes into account the measured solar wind speed and the relative distances and positions of the sun, Saturn, and the spacecraft, respectively, at any instant point of time. The propagation times of solar wind features to move over the spacecraft-Saturn distance decrease from 5.5 days, at the beginning of the analysis interval, down to a few hours just before the Voyager 2-Saturn closest approach. Each ram pressure value represents an average over one Saturn rotation period, and is chosen out of 10.66-hours running averages to be closest in time to the corresponding SKR energy value.

The bars in the second panel indicate the periods of low solar wind plasma density events, which evidently were spacecraft encounters with the Jupiter magnetotail. This 
phenomenon and its implications concerning this investigation will be discussed in the subsequent section.

The third panel of Fig. 1 shows the interplanetary magnetic field magnitude. These data points are treated in the same manner as the ram pressure values, representing 10.66 hours averages ballistically projected from the spacecraft to Saturn.

The three quantities shown in the three panels of Fig. 1 represent each an experiment onboard Voyager 2. (For detailed descriptions of the PRA-, PLS-, and MAG-experiment see, respectively, WARWICK et al. (1977), BRIDGE et al. (1977), and BEHANNON et al. (1977).)

The range of variation of these three quantities is different: Values as low as $0.04 \times 10^{12}$ Joules per steradian for the isotropic SKR energy are followed by increases up to $1.15 \times 10^{13}$ Joules per steradian indicating changes of more than two orders of magnitude within time scales of a few Saturn rotations. The same range of variation (factor 100) can be observed in the solar wind ram pressure within comparable time scales. Finally the IMF magnitude varies less distinctly over only about one order of magnitude, but also with time scales similar to the SKR energy and the solar wind ram pressure.

These variations occur during "normal" solar wind conditions, i.e. during times of no Jupiter magnetotail contamination. As explained below the solar wind and interplanetary magnetic field conditions are distinctly different from those within the extended Jovian magnetotail. Therefore any study on the influence of the solar wind and IMF on the Saturnian kilometric radiation must carefully distinguish between these different domains.

\section{Jovian Magnetotail Encounters}

The predictions of encountering Jupiter's distant magnetotail by the Voyager 2 spacecraft during the approach to Saturn were confirmed by a number of papers (SCARF et al., 1981; KURTH et al., 1982; LEPPING et al., 1982, 1983), which brought some insight into a new and fascinating phenomenon: the existence of a Jovian magnetic structure extending from Jupiter to Saturn and maybe beyond.

There are several indicators favoring the interpretation of the Voyager 2 measurements as Jovian magnetotail encounters (KURTH et al., 1982). First, the geometry of trajectories of both the planet Jupiter and the spacecraft implies a high probability of encounters with the aberrated magnetotail. Then the encounter periods are characterized by low plasma density intervals indicating a shielding off the normal solar wind conditions by the tail magnetic field. Third, the measured magnetic field orientation coincides in general with the expected Jovian tail direction. As a fourth argument KURTH et al. (1982) pointed out the appearance of Jovian continuum radiation guided along the low-density tail region. This list can be completed by a further argument provided by DESCH (1983), who found repeated decreases in the emitted intensity of SKR as a consequence of Saturn immersions in the Jovian magnetic tail. The interpretation of this fact is given later on.

The low plasma density periods are considered to be the most significant indicator for Jupiter tail encounter intervals. According to the definitions set by KURTH et al. (1982) the "overall" low density event is determined during which the density remained below $0.05 \mathrm{~cm}^{-3}$. Within such an event a so-called "tail" event is classified by a density 
below $0.03 \mathrm{~cm}^{-3}$ over at least 6 hours, and a "core" event requires a density below 0.01 $\mathrm{cm}^{-3}$.

Within the frame of these definitions the aforementioned bars in the second panel of Fig. 1 indicate periods of tail events as recorded by the plasma science experiment onboard Voyager 2. For reasons of consistency these periods are also ballistically propagated from the point of observation to the position of Saturn.

Figures 2 and 3 are expanded plots for the intervals from day of year (DOY) 60 through 100, and from DOY 160 through 200, 1981, respectively, as shown in Fig. 1. The blow-up visualizes the dominant effect of the Jovian magnetotail on quantities like the solar wind ram pressure and the interplanetary magnetic field magnitude. In the case of normal solar wind conditions the ram pressure reaches values of more than $2.0 \times 10^{-10} \mathrm{~Pa}$, whereas during tail event periods the pressure remains below a level of $0.3 \times 10^{-10} \mathrm{~Pa}$. A similar behavior-high values during "normal" solar wind conditions and low values during Jovian magnetotail encounter periods-can be seen in the corresponding panels for the IMF. The range of variation of the IMF during the overall period (Fig. 1) is from less than 0.1 gamma $\left(10^{-10}\right.$ Tesla) to 3.2 gamma, whereas during Jovian tail encounter intervals the level of 0.9 gamma is not exceeded.

During the particular tail event interval from DOY 91 through 103, 1981, which is the actual spacecraft event time (SCET), Voyager 2 was closest to the nominal aberrated tail position. This period is partially shown in Fig. 2, but time shifted according to the solar wind propagation time between the spacecraft and the interaction point at Saturn. Therefore the bars in the second panel are placed in such a way as the solar wind and the IMF structures, changed by the Jovian tail conditions, or even the Jupiter tail itself
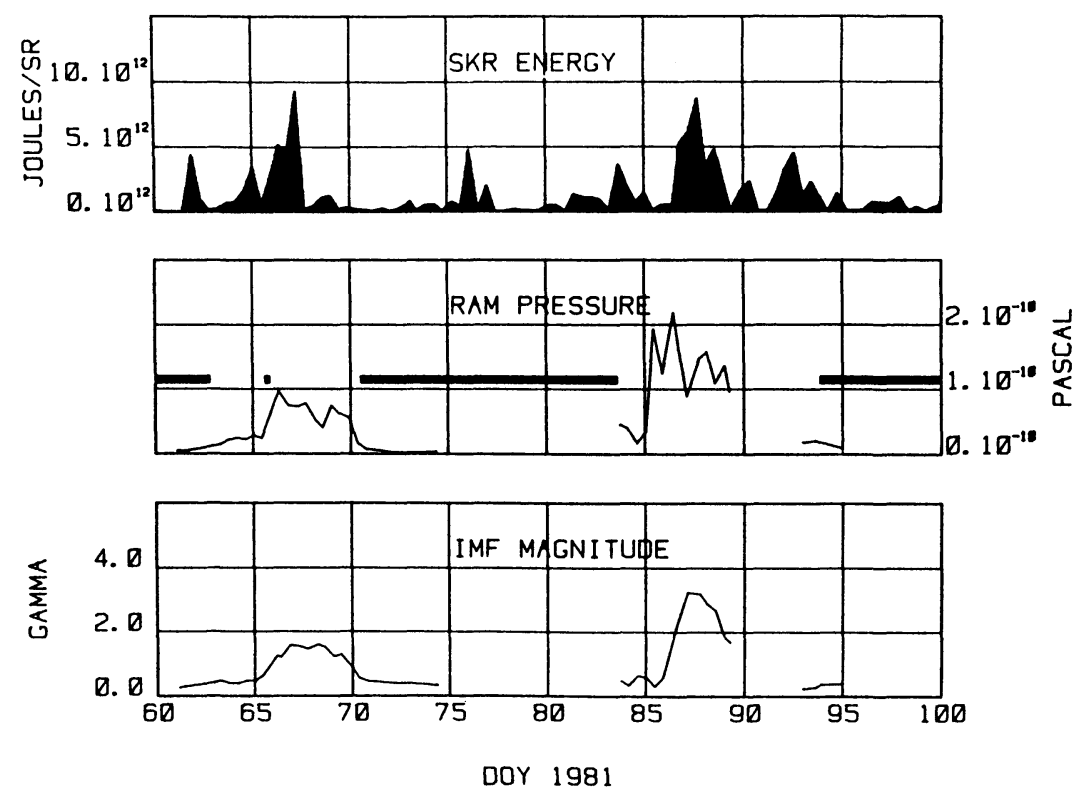

Fig. 2. Blow-up plot of data shown in Fig. 1 illustrating the enhancements of the solar wind parameters and the SKR response. Note the decrease of the ram pressure and the IMF magnitude during the Jovian magnetotail encounter periods indicated by the bars in the second panel. 

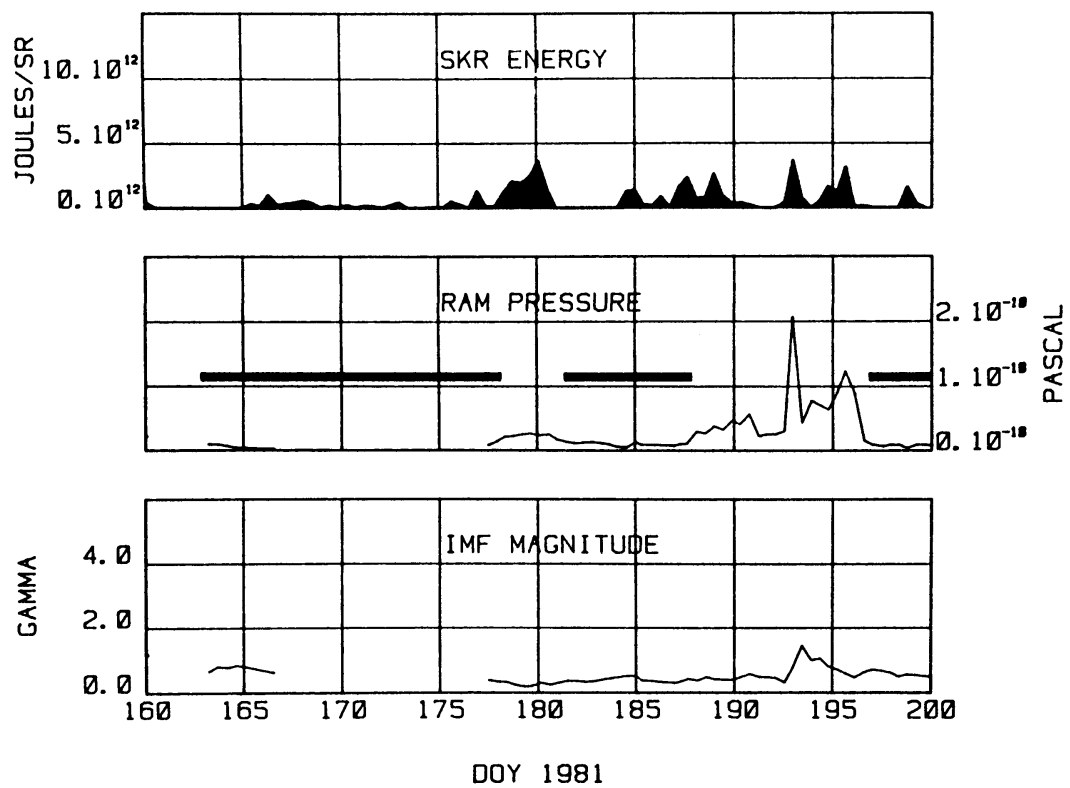

Fig. 3. Expanded plot of data shown in Fig. 1 for the interval from day 160 to 200,1981 . Note the extremely low SKR energy level during the Jovian tail encounter period from day 163 through $178,1981$.

impinge on the Saturnian magnetosphere. This phenomenon has far-reaching implications on the Saturn radio emission.

Previous investigations (DESCH, 1982; DESCH and RUCKER, 1983) have shown that SKR is strongly correlated with the solar wind ram pressure (and associated quantities like the solar wind kinetic energy flux). A further study (DESCH, 1983) found evidence of multiple Saturn immersions in the Jovian magnetotail by looking at the SKR emission level during tail encounter periods. An exceeding number of tail events saw SKR dropouts down to the Voyager 2 receiver threshold indicating a strong modulation of SKR by the solar wind plasma quantities.

As can be seen in Fig. 2 the relatively distinct SKR peaks around DOY 62 and DOY 76 , 1981, occur during tail events. But as noted by DESCH (1983) Saturn seems to be immersed by the Jupiter magnetic tail not before April 1981. During tail events following this date the emission level of SKR is decreased significantly, as shown in Fig. 3.

The purpose of this paper is to analyse the influence of the solar wind and the interplanetary magnetic field on the Saturn kilometric radiation under normal conditions, i.e. during times of no Jovian magnetotail contamination. The procedure of the applied analysis and the main outcome will be discussed in the following sections.

\section{Procedure of Analysis}

The preparation of the SKR time series is already briefly discussed in Section 2. In Fig. 1 the SKR energy values representing 1 Saturn rotation averages of the isotropic radiation energy flux are plotted at the actual spacecraft event time (SCET). However, as 
already mentioned above, the solar wind quantities (in the second and third panel of Fig. 1) are shown at the expected time of interaction at Saturn's magnetosphere, therefore these quantities are extrapolated by a ballistic propagation from the spacecraft position to Saturn. As explained in a previous paper (DESCH and RUCKER, 1983) the actual arrival times of solar wind features may depart from the predicted arrival times by about one Saturn rotation at most, which must be considered in the discussion of the results. The time difference between the actual radio emission time at Saturn and SCET even at the beginning of the interval in question does not exceed 800 seconds, which is negligible compared to the averages used in this investigation.

In order to analyse any relationship between two time series a linear cross correlation would be appropriate, but considering the frequent interruptions in the Voyager 2 data series due to the Jovian magnetotail the evidence of correlation between the solar wind and the Saturn kilometric radiation must be found by another treatment. The procedure of superposed epochs, or CHREE analysis (BELL and GLAZER, 1958; FORBUSH et al., 1983) provides a proper means in this respect.

Since the solar wind obviously can be regarded as the agent of any major modulations of Saturnian magnetospheric processes, in our case the stimulation of SKR emission, the time series of the solar wind quantities have to be checked on sudden enhancements or decreases. These significant level changes are considered as the so-called zero epochs of the CHREE analysis. This zero epoch is a characteristic and well-marked point of time within an event or within a particular period of time. In this investigation the zero epochs are determined by those points of time, where the time derivative of the solar wind time series reached or exceeded the three sigma level (with sigma as the standard deviation).

Around every zero epoch a defined time interval is established (10 Saturn rotations before and 20 rotations after any particular zero epoch) and all epochs within the solar wind time series as well as within the SKR time series (the SKR epochs of course are aligned in time) are summed up and averaged.

As a result we obtain averaged profiles, which show the relationship between quantities derived from solar wind properties and/or interplanetary magnetic field properties and the emitted SKR energy with respect to time as well as amplitude.

\section{Discussion of Results}

The study by DESCH and RUCKER (1983) on the solar wind control of the Saturn kilometric radiation already discussed in detail the solar wind quantities, which were found important in driving magnetospheric processes. The main outcome of this previous investigation using Voyager 1 data established the dominance of the solar wind ram pressure and the associated quantity, the kinetic energy flux, in stimulating the radio emission of Saturn in the kilometer wavelength band.

The present paper re-examines these findings by using Voyager 2 data and applying a different treatment due to different circumstances as mentioned in the previous sections. Figures 4 and 5 summarize six resp. eight plots, which visualize the individual relationship between the particular solar wind quantity (dashed line) and the SKR energy (full line) within a time interval of 10 Saturn rotations before and 20 rotations after the zero epoch. The data resolution for all parameters is one value per Saturn rotation. With the exception of the Akasofu parameter $\varepsilon$, which is shown in the lower right plot of Fig. 4, 

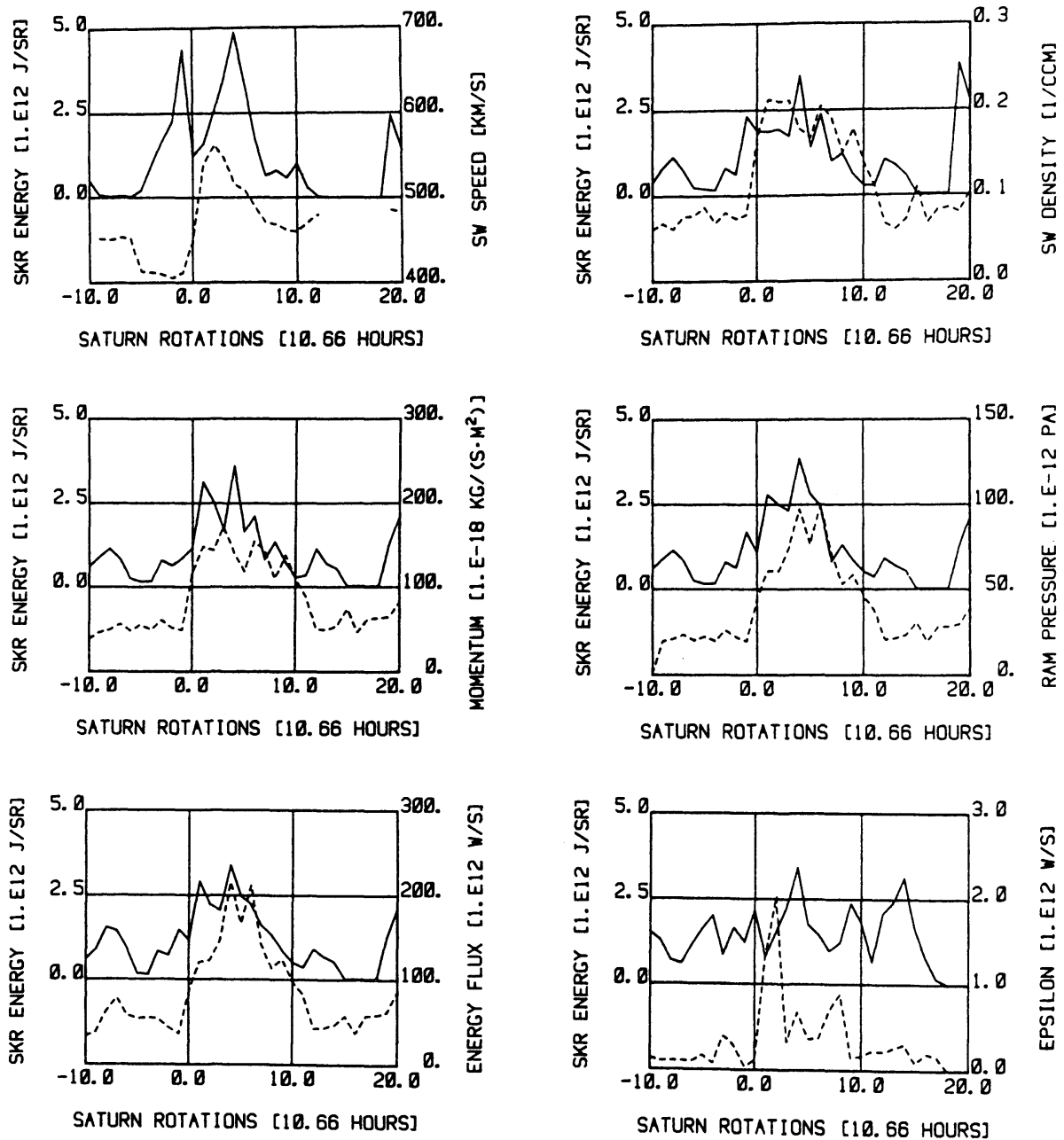

Fig. 4. Averaged profiles of SKR energy (full line) and solar wind quantities (dashed line) derived from the Voyager 2 observations during the Saturn preencounter period in 1981. Solar wind momentum, ram pressure and kinetic energy flux exhibit the most coincident course with the corresponding SKR profiles.

all other solar wind quantities of this figure can be classified as plasma parameters of the solar wind: bulk speed $V$, solar wind density $\rho$, momentum $\rho * V$, solar wind ram pressure $\rho * V^{2}$, and kinetic energy flux $\rho * V^{3}$. In Fig. 5 the eight plots throughout contain IMF properties, so the quantities shown in this figure can be classified as magnetic parameters of the solar wind: the IMF magnitude $|\boldsymbol{B}|$, the solar wind electric field $|\boldsymbol{V} \times \boldsymbol{B}|$, the quantity $V \boldsymbol{B}^{2}$ attributed to the solar wind dynamo energy flux, the Akasofu parameter $\varepsilon$, the solar wind parameter combination $B V^{2}$, the IMF- $B_{z}$ north component (aligned to the magnetic dipole axis of Saturn) and finally a measure of IMF sector boundary passages, $B_{y}$ (toward and away from the sun).

Before going into details in analysing the plots one general aspect should be addressed in brief. A close relationship between any solar wind quantity and the SKR 

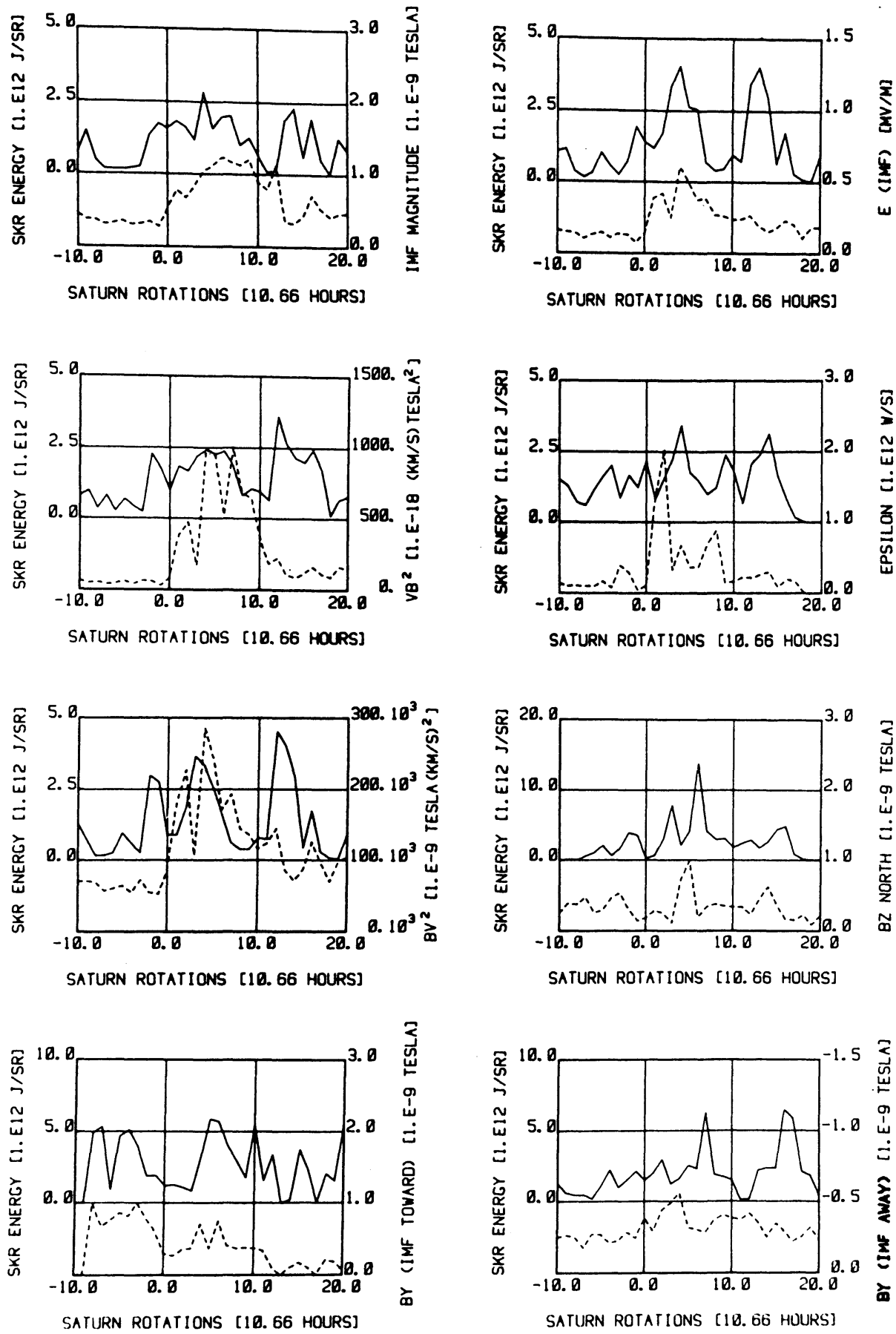

Fig. 5. Averaged profiles of SKR energy (full line) and solar wind quantities (dashed line) incorporating magnetic field properties. None of these quantities or combinations yield the profile coincidence as obtained with solar wind momentum, ram pressure and energy flux. 
energy can be expected, if any amplitude change of the cause (=the solar wind) is coincident with or followed by a corresponding modulation of the effect (=the Saturnian radiation) within reasonable, i.e. physically realizable, lag times. As demonstrated by DESCH and RUCKER (1983) possible time uncertainties due to the ballistic propagation of any solar wind features from the spacecraft position to Saturn remain within a time interval of one Saturn rotation at most. Additionally, the characteristic response time of Saturn's magnetosphere to sudden changes in the solar wind ram pressure, e.g., is found to be approximately 6 hours (NESS et al., 1982). Peak-to-peak lag times exceeding one Saturn rotation therefore cannot be attributed to the propagation procedure and should be considered as non-physical.

The upper left plot of Fig. 4 shows the relationship between the solar wind bulk velocity and the SKR energy. Aligned in time to the speed enhancement there are two SKR peaks. The first SKR peak occurs 3 Saturn rotations before, the second 2 rotations after the speed peak, and none of them reaches even the $2 \sigma$-level ( $\sigma=$ standard deviation) above the SKR mean.

The solar wind speed, which correlates best with the terrestrial kilometric radiation (GALLAGHER and D'ANGELO, 1981) can again be excluded as a quantity stimulating the SKR.

The following four solar wind quantities density, momentum, ram pressure, and kinetic energy flux have the structure $\rho V^{n}$ with $0 \leq n \leq 3$. These plasma parameters exhibit the most significant relationship to the SKR energy. The corresponding SKR peaks reach or even exceed the $3 \sigma$-level above the respective SKR means. However, the criterion regarding the lag times excludes the solar wind density: The upper right plot of Fig. 4 shows a peak-to-peak lag time of 3 Saturn rotations between the density and the SKR energy.

The plots for the momentum, ram pressure and energy flux visualize a relationship between these solar wind quantities (dashed lines) and the emitted radio energy of the SKR (full lines), which is unrivalled by any other quantity in Figs. 4 and 5. This significant relationship is evident by an almost one-to-one correspondence between the dashed and full lines over several Saturn rotations. It is interesting that the quantities $\rho V^{n}, 1 \leq n \leq 3$, stimulate a main SKR peak (reaching or exceeding the $99 \%$ confidence level), which always occurs four Saturn rotations after the zero epoch. Only the solar wind ram pressure and the kinetic energy flux have main peaks, which correspond to the main SKR peak within zero Saturn rotation lag times.

This is a remarkable confirmation of the results obtained by the Voyager 1 investigation (DESCH and RUCKER, 1983), but is obvious due to the preparation procedures, which extrude the normal solar wind conditions from the Voyager 2 observations.

Switching from mere solar wind plasma parameters to magnetic parameters or parameter combinations, the lower right plot of Fig. 4 shows the so-called Akasofuparameter $\varepsilon$. This parameter was introduced by PERREAULT and AKASOFU (1978) and can be regarded as the solar wind dynamo energy flux. At this point the $\varepsilon$-plot should only be placed in contrast to the solar wind kinetic energy flux (lower left plot of Fig. 4), a more detailed description follows below.

The upper left plot of Fig. 5 contains the interplanetary magnetic field magnitude $|\boldsymbol{B}|$. Surprisingly enough the expected relationship between $B$-enhancements and SKR energy increases, which is visible in Fig. 1 particularly around DOY 67, DOY 87, and 
DOY 157, 1981, respectively, is not confirmed in the averaged profile of $|\boldsymbol{B}|$ in Fig. 5. The SKR energy contour shows an increase already 2 Saturn rotations before the $B$ enhancement, which violates the physical causality, and the SKR peak is only about $2 \sigma$ above the SKR mean level.

The upper right plot of Fig. 5 shows the electric field $|\boldsymbol{V} \times \boldsymbol{B}|$ in the solar wind. There is a peak-to-peak correspondence between $E$ and the first SKR peak, scarcely exceeding the $2 \sigma$-level, but no correspondence between $E$ and the second SKR peak.

The subsequent plots in the second and third line of Fig. 5 show parameter combinations, which are related among themselves. The already mentioned Akasofuparameter $\varepsilon$ represents an interplanetary Poynting vector multiplied by a function, which incorporates the colatitudinal angle of the interplanetary magnetic field (KAN et al., 1980; AKASOFU, 1981, 1983; MURAYAMA, 1982). This parameter $\varepsilon$ depends upon the IMF angle in the plane containing the magnetic axis of the respective planet. Since the Akasofu-parameter was introduced for the earth, it has to be redefined for Saturn due to the reversed magnetic axis. The Akasofu parameter $\varepsilon$ predicts strong solar windmagnetosphere interaction for northward IMF and weak interaction for southward IMF. As can be seen from the $\varepsilon$-plot (right plot in the second line of Fig. 5) the involved rectifier model does not work in the case of stimulating SKR. A steep increase of $\varepsilon$ is followed by a small SKR peak within 2 Saturn rotations lag time.

The Akasofu parameter as an energy coupling function contains the term $V B^{2}$, which is separately plotted in Fig. 5 (second line left). Bare of the IMF direction this parameter combination shows neither a temporal nor an amplitudinal relationship to SKR.

An interesting feature shows up in the plot for the $B_{z}$-north component of the IMF (right plot in the third line of Fig. 5). Aligned to the direction of the Saturnian magnetic axis the IMF $B_{z}$-north component is counted if $B_{z}>0$. As previously suggested a northward IMF would facilitate possible energy coupling processes, but mainly around the Saturnian subsolar magnetopause region, which is far off the SKR source region. The $B_{z}$-north plot of Fig. 5 shows a magnetic peak followed by an SKR peak within one Saturn rotation. Note the change in the SKR energy scale. The significance of any inherent relationship, if it is, between $B_{z}$-north and SKR is diminished by the fact that a sufficient number of epochs could only be found by lessening the level to $2 \sigma$ for the zero epoch determination.

No evidence of any association between $B_{z}$-south and SKR is found, a corresponding plot is omitted. The same vague situation is found in the relation between the quantity $B V^{2}$ and SKR (left plot in the third line of Fig. 5).

The importance of the interplanetary magnetic field, especially the $B_{z}$-component, in controlling magnetospheric processes is evident and documented in numerous papers. But also the $B_{y}$-component, in particular the IMF sector boundary transitions, can affect the planetary magnetic activity and related phenomena (CANDIDI et al., 1983; YOSHIZAWA, 1984). Therefore a final approach is taken to how the IMF sector polarity might influence the Saturnian kilometric radiation.

At the orbit of Saturn sector boundary transitions of the IMF are mainly indicated by a change of the $B_{y}$-component within the frame of the spacecraft coordinate system ( $\boldsymbol{R}=$ sun-spacecraft radial direction, $\boldsymbol{T}=$ azimuthal direction aligned to the Saturnian orbital motion=positive $B_{y}, \boldsymbol{N}=$ perpendicular to $\boldsymbol{R}$ and $\boldsymbol{T}$ in a right-hand system). The theoretical orientation of the IMF spiral structure (HUNDHAUSEN, 1972) was essentially 
confirmed by the Voyager 2 measurements by establishing histograms of the azimuthal IMF-angles. The classes of $B_{y}$ (toward the sun) and $B_{y}$ (away from the sun) could be found easily. Shortening the outcome no relationship could be found between the IMF sector boundary transitions and the SKR energy, as can be seen in the last 2 plots of Fig. 5.

\section{Conclusion}

Under normal solar wind conditions, which comprehend the quiet solar wind as well as high speed streams, the Saturn kilometric radiation is highly modulated and controlled by the fluctuations of the solar wind plasma quantities $\rho V^{n}(1 \leq n \leq 3)$, with a slightly better correlation of solar wind ram pressure and energy flux than momentum. No significant relationship to SKR is found with quantities incorporating the magnetic property of the interplanetary medium. This fact does not exclude the possibility that magnetic parameters might have some influence on the generation and beaming of SKR at timescales shorter than 1 Saturn rotation.

This Voyager 2 investigation confirms the results found so far by the Voyager 1 observations.

In this study no mention is made upon the involved energetics, the energy transfer from the solar wind into the Saturnian magnetosphere and its conversion to radio power. Recent papers on new energy coupling functions might provide further insight in this topic, which is beyond the scope of this investigation.

We are especially grateful to N. F. Ness, Voyager Magnetometer team principal investigator, and to H. S. Bridge, Plasma Science team principal investigator, for making the necessary data available for this study. We thank G. K. F. Rabl for the assistance in preparing the figures.

\section{REFERENCES}

AKasofu, S.-I., Energy coupling between the solar wind and the magnetosphere, Space Sci. Rev., 28, $121,1981$. AKAsofu, S.-I., Solar-wind disturbances and the solar wind-magnetosphere energy coupling function, Space Sci. Rev., 34, 173, 1983.

Barrow, C. H., Decametre-wave radiation from Jupiter and solar activity, Planet. Space Sci., 20, $2051,1972$.

Barrow, C. H., Jupiter's decametric radio emission and solar activity, Planet. Space Sci., 26, 1193, 1978.

BARRow, C. H., Association of corotating magnetic sector structure with Jupiter's decameter-wave radio emission, J. Geophys. Res., 84, 5366, 1979.

Behannon, K. W., M. H. Acuna, L. F. Burlaga, R. P. Lepping, N. F. Ness, and F. M. Neubauer, Magnetic field experiment for Voyagers 1 and 2, Space Sci. Rev., 21, 235, 1977.

Bell, B. and H. Glazer, Sunspots and geomagnetism, smithsonian contr, Astrophys., 2, 161, 1958.

Bridge, H. S., J. W. Belcher, R. J. Butler, A. J. Lazarus, A. M. Mavretic, J. D. Sullivan, G. L. Siscoe, and V. M. Vasyliunas, The plasma experiment on the 1977 Voyager mission, Space Sci. Rev., 21, 259, 1977.

Candidi, M., H. W. Kroehl, and C.-I. MenG, Intensity distribution of dayside polar soft electron precipitation and the IMF, Planet. Space Sci., 31, 489, 1983.

Conseil, L., Y. Leblanc, G. Antonini, and D. Quemada, The effect of the solar wind velocity in the Jovian decametric emission, Astrophys. Lett., 8, 133, 1971.

DesCh, M. D., Evidence for solar wind control of Saturn radio emission, J. Geophys. Res., 87, 4549, 1982.

DeSCH, M. D., Radio emission signature of Saturn immersions in Jupiter's magnetic tail, J. Geophys. Res., 88, $6904,1983$.

DeSCH, M. D. and C. H. BARROw, Direct evidence for solar wind control of Jupiter's hectometer wavelength radio emission, J. Geophys. Res., 89, 6819, 1984. 
Desch, M. D. and H. O. RuCKer, The relationship between Saturn kilometric radiation and the solar wind, $J$. Geophys. Res., 88, 8999, 1983.

Desch, M. D. and H. O. RuCKER, Saturn radio emission and the solar wind: Voyager 2 studies, COSPAR proceedings, Advances in Space Research, 5, 333, 1985.

Forbush, S. E., M. A. Pomerantz, S. P. Duggal, and C. H. Tsao, Statistical considerations in the analysis of solar oscillation data by the superposed epoch method, Solar Phys., 82, 113, 1983.

Gallagher, D. L. and N. D'Angelo, Correlations between solar wind parameters and auroral kilometric radiation intensity, Geophys. Res. Lett., 8, 1087, 1981.

Green, J. L., D. A. Gurnett, and R. A. Hoffman, A correlation between auroral kilometric radiation and inverted $V$ electron precipitation, J. Geophys. Res., 84, 5216, 1979.

Green, J. L., V. A. Saflekos, D. A. Gurnett, and T. A. Potemra, A correlation between auroral kilometric radiation and field-aligned currents, J. Geophys. Res., 87, 10463, 1982.

Hundhausen, A. J., Coronal Expansion and Solar Wind, Physics and Chemistry in Space Series, 5, Springer, Berlin, Heidelberg, New York, 1972.

Kaiser, M. L. and J. K. AleXAnder, Relationship between auroral substorms and the occurrence of terrestrial kilometric radiation, J. Geophys. Res., 82, 5283, 1977.

Kaiser, M. L. and M. D. DeSCH, Saturnian kilometric radiation: Source locations, J. Geophys. Res., 87, 4555, 1982.

Kaiser, M. L., J. E. P. Connerney, and M. D. Desch, Atmospheric storm explanation of Saturnian electrostatic discharges, Nature, 303, 50, 1983.

Kaiser, M. L., M. D. Desch, W. S. Kurth, A. Lecacheux, F. Genova, B. M. Petersen, and D. R. Evans, Saturn as a radio source, in Saturn, edited by T. Gehrels and M. S. Matthews, 378 pp., The University of Arizona Press, Tucson, Arizona, 1984.

KAN, J. R., L. C. LEE, and S.-I. AKASOFU, The energy coupling function and the power generated by the solar wind-magnetosphere dynamo, Planet. Space Sci., 28, 823, 1980.

Kovalenko, V. A., The relation of Jupiter's decameter radio emission to solar and geomagnetic activity, Sov. Astron., 15, 478, 1971.

Kurth, W. S., J. D. Sullivan, D. A. Gurnett, F. L. Scarf, H. S. Bridge, and E. C. Sittler, Jr., Observations of Jupiter's distant magnetotail and wake, J. Geophys. Res., 87, 10373, 1982.

Lecacheux, A. and F. Genova, Source localization of Saturn kilometric radio-emission, J. Geophys. Res., 88, 8993, 1983.

Lepping, R. P., L. F. Burlaga, M. D. Desch, and L. W. Klein, Evidence for a distant $\left(>8700 \mathrm{R}_{\mathrm{j}}\right)$ Jovian magnetotail: Voyager 2 observations, Geophys. Res. Lett., 9, 885, 1982.

Lepping, R. P., M. D. Desch, L. W. Klein, E. C. Sittler, Jr., J. D. Sullivan, W. S. Kurth, and K. W. BehanNon, Structure and other properties of Jupiter's distant magnetotail, J. Geophys. Res., 88, 8801, 1983.

Murayama, T., Coupling function between solar wind parameters and geomagnetic indices, Rev. Geophys. Space Phys., 20, 623, 1982.

Ness, N. F., M. H. Acuna, K. W. Behannon, L. F. Burlaga, J. E. P. Connerney, R. P. Lepping, and F. M. Neubauer, Magnetic field studies by Voyager 2: Preliminary results at Saturn, Science, 215, 558, 1982.

OYA, H. and A. MORIOKA, Effect of turbulent region of interplanetary magnetic field on Jovian decametric radio emissions from the main source (abstract), EOS Trans. AGU, 58, 757, 1977.

Perreault, P. and S.-I. Akasofu, A study of geomagnetic storms, Geophys. J. R. Astron. Soc., 54, 547, 1978.

Rucker, H. O. and M. D. Desch, External control of planetary radio emission, Proc. Austrian Acad. Sci., 192, 433, 1983.

Rucker, H. O., G. K. F. RABL, and M. D. Desch, External control of the Saturn kilometric radiation by the solar wind: Comparison between Voyager 1 and 2 observations, Ann. Geophys., 7, 341, 1989.

Scarf, F. L., W. S. Kurth, D. A. Gurnett, H. S. Bridge, and J. D. Sullivan, Jupiter tail phenomena upstream from Saturn, Nature, 292, 585, 1981.

Terasawa, T., K. Maezawa, and S. Machida, Solar wind effect on Jupiter's non-Io related radio emission, Nature, 273, 131, 1978.

Warwick, J. W., J. B. Pearce, R. G. Peltzer, and A. C. Riddle, Planetary radio astronomy experiment for Voyager missions, Space Sci. Rev., 21, 309, 1977.

YoshizAWA, K., Influence of IMF-sector polarity on the north-south asymmetry of geomagnetic activity and its relationship with solar wind parameters, Planet. Space Sci., 32, 605, 1984. 